\title{
Exploration of the Scientific Research Ability Cultivation of Major Basic Course Teachers in Military Science and Technology Universities
}

\author{
Jingna Cui, Jian Pan, Xinwei Gao, Luya Zhang, Shasha Wu \\ Test Training Base of Information and Communication School, National University of Defense \\ Technology, Xi'an 710106, China
}

\begin{abstract}
Scientific research is an important function of modern colleges and universities, for teachers who undertake major basic courses, they has undertaken basic teaching for a long time, their scientific research development has been constrained, which are mainly expressed in the following aspects: weak scientific research consciousness, weak scientific research ability, weak scientific research atmosphere, and insufficient scientific research impetus, so it is very necessary to improve their scientific research ability. On the basis of this current situation, this paper proposes to build "strengthen awareness-rational position-training platform-evaluation mechanism" four-in-one ability cultivation system.
\end{abstract}

Keywords: Major Basis; Scientific Research Ability; Scientific Research and Teaching.

\section{Introduction}

The level of technological innovation and scientific research development of colleges and universities is related to the school-running quality and benefit of colleges and universities, in particular, high-level scientific research results often represent the academic status and influence of the corresponding disciplines of the school. The level of scientific research ability of academics is an important factor for the personal professional development of teachers and the sustainable development of universities.

For professional colleges, disciplines have strong characteristics. Taking this unit as an example, it is mainly responsible for the task of cultivating junior communication command military talent for the whole army, all communications major non-commissioned officers are required to offer major basic courses-electronic technology and communication principles courses before in-depth study of professional knowledge.

In order to save teaching resources, the school has set up the major basic course's implementation group in the basic teaching department. But at the same time, it also brings some problems, since it is separated from various departments in administrative relations, moreover, it faces the current situation of drastic reduction in class hours, and the scientific research state of teachers of major basic courses often has the following characteristics:

Firstly, their scientific research awareness is weak. Because they are in the basic teaching department, no matter in the input of teaching resources, guarantee of teaching conditions, or the construction of course culture, none of them has formed the overall atmosphere and teaching culture such as major and discipline construction.

Secondly, their scientific research ability is not strong, and their scientific research level urgently needs to be improved. (1) The contents which are involved in major basic course teaching are relatively mature, cannot keep up with cutting-edge knowledge, it is difficult to innovate from their own major. (2) Basic courses and major courses belong to different units, relatively speaking, and basic courses and major courses are relatively weakly related. Taking this teaching and research office as an example, influenced by the teaching-based atmosphere for many years, most teachers of major basic courses are just beginning to participate in teaching and research projects, and rarely participate in scientific research projects. (3) Participation in scientific research is only for promotion of professional titles, they are eager for quick success and instant benefits in the application of scientific research projects and paper publication, they do not consider the improvement of scientific research 
ability and personal teaching ability, as a result, their scientific research directions swing, the level of scientific research results is low, and the improvement of teaching quality is limited.

Thirdly, the scientific research atmosphere is not strong, and the scientific research software is poor. At present, the scientific research work is basically "lone wolf", there is no scientific research team, or the enthusiasm of the scientific research team has not been motivated, and there is a lack of substantive cooperation, for example, the process of subject declaration-research-form scientific research results-conclusion is usually completed by the subject leader alone, resulting in dispersed scientific research resources and weak joint forces.

Fourthly, the motivation of scientific research is not enough, quantitative management methods of scientific research performance are deficient, which affect the enthusiasm of teachers to engage in scientific research to a certain extent, and objectively restricts teachers from achieving high-level scientific research results.

Scientific research is an important function of modern colleges and universities, and it is very necessary for teachers who specially undertake basic professional courses to improve their scientific research ability. In allusion to the analysis of the scientific research state of major basic teachers, it plans to build "strengthen awareness-rational position-training platform-evaluation mechanism" fourin-one ability training system.

\section{Strengthen Scientific Research Awareness and Set Correct Scientific Research Value}

The recognition of the importance of scientific research is the first step in strengthening scientific research ability. During the implementation, it plans to cooperate with the school's scientific research management department, jointly carry out the following activities with the teaching and research office: firstly, organize and study the "Notice on the Development of New Engineering Research and Practice", the "Notice on the Promotion of New Engineering Research and Practice Projects" and other documents, so that the teachers' understanding for scientific research rise to a new height.

Secondly, carry out the "change scientific research concepts and unify scientific research understanding" discussion activities, listen to the various ideas of teachers on scientific research, help teachers clean up the relationship between teaching and scientific research through discussions, to make it unified and developed equally. Finally, the guidance and support activities for teachers must be continuous, and the cultivation and transformation of scientific research concepts requires a process, it is a gradual process from "not focusing on scientific research" to "focusing on scientific research", from "initial stage" to "intermediate and advanced stage", make sure they avoid the impetuous mentality of seeking quick success and instant benefits.

\section{Reasonable Position and Clear Scientific Research Orientation}

Choice is more important than hard work, as far as schools, departments, and teachers themselves are concerned, establishing the clear scientific research position is the most important link of improving scientific research efficiency. Establishing the reality of professional technology development, it plans to collect opinions widely across the department, organize leaders, experts, and teaching team leaders to discuss, establish research directions, insist on doing something need to do and not doing something. Serving the improvement of its own education, teaching and management level as rallying point, the research on topics such as education, teaching and management is actively carried out; strengthening the cooperation among army, enterprises and colleges as rallying point, research in applied technology is actively carried out. In some basic theoretical research and major scientific and technological research projects, there is nothing to be done. The topic will be further refined to improve the scientific research ability of teachers from two directions: basic research and applied research. 


\section{1). Basic Research}

Teaching and research projects continue to advance steadily. The knowledge structure is relatively stable, and various teachers teach one course, etc., which exists in major basic courses, the teachers set groups, regularly exchange and discuss teaching reflections, study new teaching ideas and concepts, and then apply for quality courses, write excellent course textbooks, and apply for educational reform project. After the staged research results are available, the teachers can apply for the National Natural Science Foundation of China, and carry out model simulation verification in combination with engineering problems.

\section{2). Applied Research}

It is not easy for teachers of major basic courses to directly find breakthroughs in scientific research from themselves; they can combine the characteristics of the school major settings, find the scientific research direction suitable for them for their major condition. It plans to strengthen from the following three points:

(1) Rely on the characteristics of this discipline and strengthen the connection with the professional field.

On the one hand, the integration and development paths of major basic course teachers and major departments are explored, and the effective integration of major basic courses and major courses is realized: (1)the integration of information technology and courses is realized through counterpart experiment cases, and promote teaching methods with the application of simulation experiments; (2) the teaching contents are actively integrated and expanded with the professional direction of noncommissioned officers' graduation design through research. Then major basic teachers are gradually integrated into the major construction teaching team, forming major collaboration team, and jointly study the reverse reconstruction of training goals, graduation requirements and talent training programs. On the other hand, under the background of the new engineering discipline of the strong army, the boundaries among disciplines are becoming more and more blurred, and the mutual influence and cross-integration among disciplines are more obvious. Under this background, strengthen the ties with other departments, colleges, schools, enterprises, and army, look for opportunities, give full play to the characteristics of their major, and integrate personal professional advantages into scientific research work in other fields. For example, the teachers of electronic technology course can provide hardware support in other projects.

(2) Optimize the job setting and build the reasonable scientific research echelon

The scientific research innovation team is the key to enhance the school's scientific research strength and promote the connotation development of the school. Relying on the school's development position, its own characteristics and major advantages, and make clear the team building goals. In the specific construction process of the subject, according to the reasonable academic relationship structure and age structure, the grassroots academic organization is composed of people who mutually accept responsibility for a common goal, and have certain expertise in various professional fields Give full play to the individual abilities of each teacher, and conduct scientific research through the power of the group. On the one hand, the scientific research support model of help, instruction and guidance among "experts and newcomers" is formed, the good scientific research team is built, and the good atmosphere where everyone engages in scientific research and everyone participates in scientific research is gradually formed. Pay attention to the cultivation of leaders, give full play to the leading role of project leaders, chief experts and professors, and strengthen the responsibilities of chief experts and professors of project leaders. Take the project as the link, form the scientific research echelon, and increase team building. On the other hand, according to the classification and review method of our school's professional titles, the original job system of major basic teachers can be optimized, and major technical jobs can be divided into teaching jobs and teaching scientific research jobs, the teaching post mainly focuses on teaching, the responsibilities of teaching and research jobs are to pay equal attention to teaching and research. The class hours have been greatly reduced through the scientific research jobs, let teachers with strong 
teaching and scientific research ability have more time to conduct scientific research and achieve results as soon as possible.

(3) Actively participate in various teaching and innovation competitions, and promote research through competitions

By participating in teaching competitions, competitions are used to promote teaching, teaching is used to promote research, and teaching and research reinforce each other. The engineering practice and innovative ability are improved by instructing non-commissioned officers to participate in innovative competitions.

\section{Build Training Platform and Create Good Scientific Research Environment}

On the basis of the existing teacher cultivation service department, the organizational structure is further improved and the division of responsibilities is made clear for major basic teachers, including the formulation of training plans, training process supervision, consulting services, etc., formulation of relevant training mechanism incentives, and supporting teachers to consciously improve their own scientific research ability.

The scientific research information exchange platform creates conditions for teachers to strengthen communication with other departments, fraternal colleges and enterprises, such as holding regular academic exchange meetings, inviting enterprises, industry experts, and scholars to discuss and exchange the latest industry development trends with teachers. Moreover, it provides teachers' opportunities for academic exchanges with the outside world. Relevant departments should propose various training implementation methods, and make corresponding training content based on different ages, different teaching years, and different professional titles, etc.

The applied technology research platform is built by each scientific research team. On the one hand, its own scientific research path is promoted by relying on the integration plan between major foundations and major courses. On the other hand, upholding the principle of valuing innovation but not only innovation, regularly organizes members to share scientific research inspiration and ideas, discuss the experience and difficulties in the research process, and provide a certain reference for the next scientific research work. Moreover, each team strengthens the integration of related resources (including human, financial, material, etc.), and form complementary advantages.

The scientific research business guidance platform mainly provides guidance work for teachers to master work processes and basic requirements to scientific research.

In addition to the relevant management departments, the moderate part-time management personnel are made clear in the teaching and research office and scientific research team.

\section{Establish the Scientific Performance Evaluation and Incentive Mechanism}

In order to promote the stable development of scientific research work, ensure that the enthusiasm of major basic teachers is fully mobilized, and the scientific research evaluation and incentive mechanism are adapted to the new situation of scientific research development.

Follow multi-level, multi-dimensional, multi-index classification to comprehensively improve the evaluation mechanism:

The targeted evaluation standards are set. According to the job types of major basic teachers, such as teaching jobs, teaching and scientific research jobs, etc., the evaluation standards are different. Moreover, the equivalent conversion mechanism of scientific research workload and teaching workload is explored, and use it as one of the main bases for professional title evaluation and job promotion. Moreover, based on the scientific research incentive mechanism, the teaching competition reward method is made.

The good situation where leaders attach importance to scientific research, teachers participate in scientific research, management serve scientific research is formed via the above measures. 
Volume 3 (2021)

In short, understanding the relationship between scientific researches and teaching dialectically, schools and teachers fully understand the importance of scientific research in order to better promote the growth of major basic course teachers.

\section{References}

[1] Yu Zhihong. Research and Exploration on the Effective Integration of Major Basic Courses and Major Courses [J]. Science \& Technology Vision, 2017(16).

[2] Li Li. Exploration of the Improvement Strategies of Scientific Research Ability of Young Teachers in Higher Vocational Colleges in the Information Technology Competition [J]. Journal of Reading and Writing, 2019 (16).

[3] Feng Aihong, Luo Sheguan. Investigation and Analysis of the Status of the Professional Development of Young and Middle-Aged Teachers in Shanxi[J]. Social Sciences Journal of Universities in Shanxi, 2019 (31). 